\title{
Wide angle Compton scattering on the proton: study of power suppressed corrections
}

\author{
N. Kivel ${ }^{1,2, a}$, M. Vanderhaeghen ${ }^{1,2}$ \\ ${ }^{1}$ Helmholtz Institut Mainz, Johannes Gutenberg-Universität, 55099 Mainz, Germany \\ ${ }^{2}$ Institut für Kernphysik, Johannes Gutenberg-Universität, 55099 Mainz, Germany
}

Received: 17 June 2015 / Accepted: 22 September 2015 / Published online: 8 October 2015

(C) The Author(s) 2015. This article is published with open access at Springerlink.com

\begin{abstract}
We study the wide angle Compton scattering process on a proton within the soft-collinear factorization (SCET) framework. The main purpose of this work is to estimate the effect due to certain power suppressed corrections. We consider all possible kinematical power corrections and also include the subleading amplitudes describing the scattering with nucleon helicity flip. Under certain assumptions we present a leading-order factorization formula for these amplitudes which includes the hard- and soft-spectator contributions. We apply the formalism and perform a phenomenological analysis of the cross section and asymmetries in the wide angle Compton scattering on a proton. We assume that in the relevant kinematical region where $-t,-u>2.5 \mathrm{GeV}^{2}$ the dominant contribution is provided by the soft-spectator mechanism. The hard coefficient functions of the corresponding SCET operators are taken in the leading-order approximation. The analysis of existing cross section data shows that the contribution of the helicity-flip amplitudes to this observable is quite small and comparable with other expected theoretical uncertainties. We also show predictions for double polarization observables for which experimental information exists.
\end{abstract}

\section{Introduction}

Wide angle Compton scattering (WACS) on a proton is one of the most basic processes within the broad class of hard exclusive reactions aimed at studying the partonic structure of the nucleon. The first data for the differential cross section of this process has already been obtained long time ago [1]. New and more precise measurements were carried out at Jefferson Lab (JLab) [2]. Double polarization observables for

On leave of absence from St. Petersburg Nuclear Physics Institute, 188350, Gatchina, Russia.

\footnotetext{
a e-mail: kivel@kph.uni-mainz.de
}

a polarized photon beam and by measuring the polarization of the recoiling proton were also measured at JLab [3]. New measurements of various observables at higher energies are planned at the new JLAB $12 \mathrm{GeV}$ facility, see e.g. [4].

The asymptotic limit of the WACS cross section, as predicted by QCD factorization, has been studied in many theoretical works [5-8]. It was found that the leading-twist contribution described by the hard two-gluon exchange between three collinear quarks predicts much smaller cross sections than is observed in experiments. One of the most promising explanations of this problem is that the kinematical region of the existing data is still far away from the asymptotic limit where the hard two-gluon exchange mechanism is predicted to dominate. Hence one needs to develop an alternative theoretical approach which is more suitable for the kinematic range of existing experiments.

Several phenomenological considerations, including the large value of the asymmetry $K_{\mathrm{LL}}$ [3] indicate that the dominant contribution in the relevant kinematic range can be provided by the so-called soft-overlap mechanism. In this case the underlying quark-photon scattering is described by the handbag diagram with one active quark while the other spectator quarks are assumed to be soft. Various models have been considered in order to implement such a scattering picture within a theoretical framework: diquarks Ref. [9], GPD models [10-13] and constituent quarks [14].

An attempt to develop a systematic approach within the soft collinear effective theory (SCET) framework was discussed in Refs. $[15,16]$. The description can be considered as a natural extension of the collinear factorization to the case with soft spectators. In our previous work the factorization of the three leading power amplitudes has been studied and a phenomenological analysis was made. The three amplitudes describing Compton scattering which involve a nucleon helicity-flip are power suppressed and they were neglected in our previous analysis. In the present work we want to include these amplitudes into our description, together with all kine- 
matical power corrections. For that purpose we discuss the factorization of helicity-flip amplitudes assuming that it can be described as a sum of hard- and soft-spectator contributions. We show that the corresponding soft contributions are described by the appropriate subleading so-called SCET-I operators. As a first step toward a proof of the factorization we restrict our attention only to the relevant operators which appear in the leading-order approximation in $\alpha_{s}$. Assuming that such soft contributions are dominant we estimate their possible numerical impact on the cross section and asymmetries.

Our work is organized as follows. In Sect. 2 we briefly describe the kinematics, amplitudes, cross sections and asymmetries. In Sect. 3 we discuss the factorization scheme for the subleading amplitudes, describe the suitable SCET-I operators and their matrix elements. We also compute the corresponding leading-order coefficient functions and provide the resulting expressions for the amplitudes. Section 4 is devoted to a phenomenological analysis and in Sect. 5 we summarize our conclusions.

\section{Kinematics and observables}

In this paper we follow the notations introduced in Ref. [16]. For convenience, we briefly summarize the most important details. In our theoretical consideration we will use the Breit frame where in and out nucleons (with momenta $p$ and $p^{\prime}$, respectively) move along the $z$-axis and $p_{z}=-p_{z}^{\prime}$. Using the auxiliary light-like vectors

$n=(1,0,0,-1), \quad \bar{n}=(1,0,0,1), \quad(n \cdot \bar{n})=2$,

the light-cone expansions of the momenta can be written as follows:

$p=W \frac{\bar{n}}{2}+\frac{m^{2}}{W} \frac{n}{2}, \quad p^{\prime}=\frac{m^{2}}{W} \frac{\bar{n}}{2}+W \frac{n}{2}$,

where $m$ is nucleon mass and the convenient variable $W$ can be expressed through the momentum transfer $t$ as

$W=m\left(\sqrt{\frac{-t}{4 m^{2}}}+\sqrt{1+\frac{-t}{4 m^{2}}}\right)$.

The photon momentum reads

$q=(q \cdot n) \frac{\bar{n}}{2}+(q \cdot \bar{n}) \frac{n}{2}+q_{\perp}$,

with

$$
\begin{aligned}
& (q \cdot n)=-\frac{\left(u-m^{2}\right)+\kappa\left(s-m^{2}\right)}{W\left(1-\kappa^{2}\right)}, \\
& (q \cdot \bar{n})=\frac{s-m^{2}+\kappa\left(u-m^{2}\right)}{W\left(1-\kappa^{2}\right)},
\end{aligned}
$$

where $\kappa=m^{2} / W^{2}$. In the limit $s \sim-t \sim-u \gg m^{2}$ these expressions can be simplified neglecting the power suppressed contributions:

$$
\begin{aligned}
& p \simeq W \frac{\bar{n}}{2}, \quad p^{\prime} \simeq W \frac{n}{2}, \quad q \simeq \frac{-u}{W} \frac{\bar{n}}{2}+\frac{s}{W} \frac{n}{2}+q_{\perp}, \\
& q^{\prime} \simeq \frac{s}{W} \frac{\bar{n}}{2}+\frac{-u}{W} \frac{n}{2}+q_{\perp}
\end{aligned}
$$

where we assume that $W \simeq \sqrt{-t}$.

For the amplitude we borrow the parametrization from Ref. [17]

$$
\begin{aligned}
M^{\gamma p \rightarrow} \gamma p=-e^{2} \varepsilon^{* \mu}\left(q^{\prime}\right) \varepsilon^{\nu}(q) \bar{N}\left(p^{\prime}\right) \mathcal{A}^{\mu v} N(p), \\
\mathcal{A}^{\mu \nu}=\left\{-\mathcal{T}_{12}^{\mu \nu}\left(T_{1}+\not{K} T_{2}\right)-\mathcal{T}_{34}^{\mu \nu}\left(T_{3}+\not K T_{4}\right)\right. \\
\left.+\mathcal{T}_{5}^{\mu \nu} i \gamma_{5} T_{5}+\mathcal{T}_{6}^{\mu \nu} i \gamma_{5} \not K T_{6}\right\},
\end{aligned}
$$

where $e$ denotes the electromagnetic charge of the proton, $N(p)$ is the nucleon spinor. In Eq. (7) we introduced the orthogonal tensor structures

$$
\begin{aligned}
& \mathcal{T}_{12}^{\mu \nu}=-\frac{P^{\prime \mu} P^{\prime \nu}}{P^{\prime 2}}, \quad \mathcal{T}_{34}^{\mu \nu}=\frac{N^{\mu} N^{\nu}}{N^{2}}, \\
& \mathcal{T}_{5}^{\mu \nu}=\frac{P^{\prime \mu} N^{\nu}-P^{\prime \nu} N^{\mu}}{P^{\prime 2} K^{2}}, \quad \mathcal{T}_{6}^{\mu \nu}=\frac{P^{\prime \mu} N^{\nu}+P^{\prime \nu} N^{\mu}}{P^{\prime 2} K^{2}},
\end{aligned}
$$

with

$$
\begin{aligned}
P & =\frac{1}{2}\left(p+p^{\prime}\right), \quad K=\frac{1}{2}\left(q+q^{\prime}\right), \\
P^{\prime} & =P-K \frac{(P \cdot K)}{K^{2}}, \quad N_{\mu}=\varepsilon_{\mu \alpha \beta \gamma} P^{\alpha} \frac{1}{2}\left(p-p^{\prime}\right)^{\beta} K^{\gamma} .
\end{aligned}
$$

The scalar amplitudes $T_{i} \equiv T_{i}(s, t)$ are functions of the Mandelstam variables.

The analytical expressions for various observables can also be found in Ref. [17]. In our consideration it will be convenient to redefine two helicity-flip amplitudes as

$\bar{T}_{1}=T_{1}+\frac{m(s-u)}{4 m^{2}-t} T_{2}, \quad \bar{T}_{3}=T_{3}+\frac{m(s-u)}{4 m^{2}-t} T_{4}$,

The reason for such a redefinition will be clarified later. The cross section reads

$$
\frac{\mathrm{d} \sigma}{\mathrm{d} t}=\frac{\pi \alpha^{2}}{\left(s-m^{2}\right)^{2}} W_{00},
$$

with

$$
\begin{aligned}
W_{00}= & \frac{\left(m^{4}-u s\right)(-t)}{\left(4 m^{2}-t\right)}\left(\frac{1}{2}\left|T_{2}\right|^{2}+\frac{1}{2}\left|T_{4}\right|^{2}+\left|T_{6}\right|^{2}\right) \\
& +\frac{1}{2}\left(4 m^{2}-t\right)\left(\left|\bar{T}_{1}\right|^{2}+\left|\bar{T}_{3}\right|^{2}\right)-t\left|T_{5}\right|^{2},
\end{aligned}
$$


cf. with Eq. (3.15a) in Ref. [17]. We also describe the asymmetries which will be considered in this work. We are interested in the beam target asymmetries with circular photon polarization $(R, L)$. In the case of a longitudinally polarized nucleon target, the corresponding asymmetry $A_{\mathrm{LL}}$ reads (in c.m.s)

$A_{\mathrm{LL}}=-\frac{\sigma_{z}^{R}-\sigma_{z}^{L}}{\sigma_{z}^{R}+\sigma_{z}^{L}}=-\frac{C_{z}^{K} W_{12}^{+}+C_{z}^{Q} W_{22}^{+}}{W_{00}}$.

Two further asymmetries describe the correlations of the recoil polarization with the polarization of the photons:

$K_{\mathrm{LL}}=\frac{\sigma_{z^{\prime}}^{R}-\sigma_{z^{\prime}}^{L}}{\sigma_{z^{\prime}}^{R}+\sigma_{z^{\prime}}^{L}}=\frac{C_{z^{\prime}}^{K} W_{12}^{-}+C_{z^{\prime}}^{Q} W_{22}^{-}}{W_{00}}$,
$K_{\mathrm{LS}}=\frac{\sigma_{x^{\prime}}^{R}-\sigma_{x^{\prime}}^{L}}{\sigma_{x^{\prime}}^{R}+\sigma_{x^{\prime}}^{L}}=\frac{C_{x^{\prime}}^{K} W_{12}^{-}+C_{x^{\prime}}^{Q} W_{22}^{-}}{W_{00}}$,

where (for more details see [17])

$W_{12}^{ \pm}=\left(4 m^{2}-t\right)\left(\bar{T}_{3}-\bar{T}_{1}\right) T_{6}^{*} \pm t\left(T_{2}+T_{4}\right) T_{5}^{*}$,

$W_{22}^{ \pm}= \pm 4 m \frac{m^{4}-s u}{4 m^{2}-t}\left(T_{2}-T_{4}\right) T_{6}^{*} \pm(s-u)\left(\bar{T}_{3}-\bar{T}_{1}\right) T_{6}^{*}$

$$
-(s-u)\left(T_{2}+T_{4}\right) T_{5}^{*}-4 m\left(\bar{T}_{3}+\bar{T}_{1}\right) T_{5}^{*} .
$$

The coefficients $C_{i}^{K, Q}$ read

$$
\begin{aligned}
& C_{z}^{K}=-C_{z^{\prime}}^{K}=-\frac{s-m^{2}}{2 m}-\frac{t\left(s+m^{2}\right)}{4 m\left(s-m^{2}\right)}, \\
& C_{z}^{Q}=C_{z^{\prime}}^{Q}=-\frac{t\left(s+m^{2}\right)}{4 m\left(s-m^{2}\right)}, \\
& C_{x^{\prime}}^{K}=-C_{x^{\prime}}^{Q}=-\frac{\sqrt{-t\left(m^{4}-s u\right)}}{2\left(s-m^{2}\right)} .
\end{aligned}
$$

\section{Factorization of the subleading helicity-flip amplitudes $T_{1,3,5}$}

In Ref. [16], the factorization of the helicity-conserving amplitudes $T_{2,4,6}$ was considered in the SCET framework [18-23]. The helicity-flip amplitudes are power suppressed and were neglected. In the current paper we would like to extend the SCET analysis and also consider the subleading amplitudes $T_{1,3,5}$. Below we are using the same notation for the SCET fields and charge invariant combinations as in Ref. [16].

The factorization of the helicity-conserving amplitudes $T_{2,4,6}$ is described by the sum of the soft- and hard-spectator contributions. It is natural to expect that the same general structure also holds for the subleading amplitudes $T_{1,3,5}$.
Therefore we assume that the $T$-product of the electromagnetic currents can be presented as

$$
T\left\{J^{\mu}(x), J^{\nu}(0)\right\}=\sum \tilde{C}^{\mu \nu} * \mathcal{O}_{I}+\sum O_{n}^{(i)} * \tilde{T}^{\mu \nu} * O_{\bar{n}}^{(j)},
$$

where $\mathcal{O}_{I}$ denotes the different SCET-I operators associated with the soft-spectator contribution and $O_{n}^{(i)} * \tilde{T}^{\mu \nu} * O_{\bar{n}}^{(j)}$ describes the hard-spectator term with the collinear operators $O_{n}^{(i)} \sim \lambda^{i}$, with $\lambda \sim \sqrt{\Lambda / Q}$ a generic small parameter. The sums in (22) include all possible operators in both terms. The power counting of the hard-spectator contribution is provided by the collinear operators

$O_{n}^{(i)} * \tilde{T}^{\mu \nu} * O_{\bar{n}}^{(j)} \sim \lambda^{i+j}$

These operators are constructed from the collinear quark and gluon fields. The leading-twist operator is given by the three quark operator $O_{n}^{(6)}=\bar{\chi}_{n}^{c} \bar{\chi}_{n}^{c} \bar{\chi}_{n}^{c}$ and is of order $\lambda^{6}$ (twist3 operator). In order to describe the helicity-flip amplitudes one has to include the subleading operators of order $\lambda^{8}$ (twist4). Therefore the helicity-flip amplitudes are suppressed by at least a factor $\lambda^{14}$ while the leading power amplitudes are described by the operator $O_{n}^{(6)} * T * O_{\bar{n}}^{(6)} \sim \lambda^{12}$. The explicit calculations of the hard-spectator part in Eq. (22) is ill defined, because of end-point singularities in the collinear convolution integrals, see for instance the calculation of the form factor $F_{2}$ in Ref. [24]. Only the sum of the soft- and hardspectator contributions in Eq. (22) provides a well defined result. A mechanism of cancellation of the end-point singularities among different contributions in exclusive amplitudes have been discussed in Refs. [25-29]. In present case, in order to see such cancellation one has to consider the hard-collinear factorization for the soft-spectator contribution described by the first term on the rhs of Eq. (22). However, such an analysis is very complicated and will be not considered here. More details as regards the soft-spectator contribution can be found in Refs. $[29,30]$ where the factorization of the nucleon form factors was considered. In the case of WACS one has a different hard scattering subdiagram (handbag diagram instead of the simple vertex at the tree level) and similar SCET operators $\mathcal{O}_{I}$ describing the hard-collinear configurations. Hence using an analogy with the form factors we accept as a plausible assumption that the complete factorization formulas in SCET framework can be derived in the form of Eq. (22).

In Refs. [15,16] a description of the leading-order WACS amplitudes based on Eq. (22) has been used for a phenomenological analysis of WACS data. Results obtained in these works allow one to conclude that the soft-spectator contribution dominates over the hard-spectator one that allows one to describe existing cross section data at sufficiently large $-t$ and $-u$. In this work we suppose that similar scattering 
Fig. 1 The SCET diagram illustrating the $T$-product in Eq. (32). The black squares show the interaction vertices, dashed quark lines denote the hard-collinear and external collinear particles. The parallel $(\|)$ and transverse $(\perp)$ signs show the contractions of the appropriate hard-collinear gluon fields

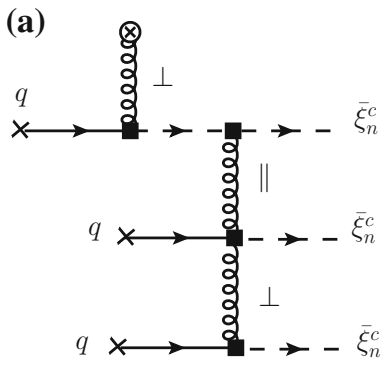

(b)

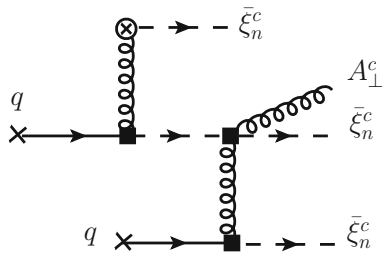

(c)

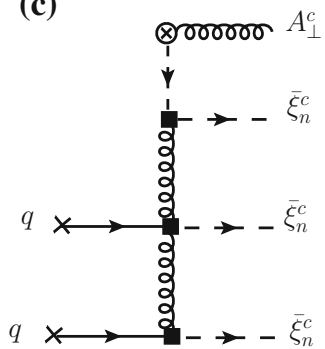

mechanism is also relevant for the subleading helicity-flip amplitudes and these amplitudes are also dominated by the soft-spectator terms. This allows us to study a contribution of such subleading corrections to various WACS observables.

The soft-spectator contribution is described by the operators $\mathcal{O}_{I}$ which are constructed from the hard-collinear fields in SCET-I. In Ref. [16] it was shown that for the leading power contribution this operator reads

$\mathcal{O}_{I}=O^{\sigma}=\sum e_{q}^{2}\left\{\bar{\chi}_{n}^{q} \gamma_{\perp}^{\sigma} \chi_{\bar{n}}^{q}-\bar{\chi}_{\bar{n}}^{q} \gamma_{\perp}^{\sigma} \chi_{n}^{q}\right\} \sim \mathcal{O}\left(\lambda^{2}\right)$.

The matrix element of this operator gives only the helicityconserving amplitudes

$\left\langle p^{\prime}\left|O^{\sigma}\right| p\right\rangle_{\text {SCET }}=\bar{N}_{n} \gamma_{\perp}^{\sigma} N_{\bar{n}} \mathcal{F}_{1}(t)$,

where

$\bar{N}_{n}=\bar{N}\left(p^{\prime}\right) \frac{\not h \hbar}{4}, \quad N_{\bar{n}}=\frac{\not h t h}{4} N(p)$.

Hence in order to describe the soft-spectator contribution of the helicity-flip amplitudes we need the subleading operators. A similar situation also holds for the proton form factors $F_{1}$ and $F_{2}$ see e.g. Ref. [30].

The matrix element of the required subleading operator must describe the chiral-odd Dirac structures appearing in the amplitudes

$\left\langle p^{\prime}\left|\mathcal{O}_{I}\right| p\right\rangle_{\mathrm{SCET}}=\bar{N}_{n} 1 N_{\bar{n}} A+\bar{N}_{n} i \gamma_{5} N_{\bar{n}} B$,

where $A$ and $B$ are some scalar SCET-I amplitudes. From Eq. (27) it follows that the SCET operator $\mathcal{O}_{I}$ can only have an even number of the transverse Lorentz indices.

The simplest operator with the required structure that can be built from the gluon fields and is of order $\lambda^{2}$ :

$O_{\mu \nu}^{(2)}=\mathcal{A}_{\perp \mu}^{n} \mathcal{A}_{\perp \nu}^{\bar{n}}+\mathcal{A}_{\perp \nu}^{n} \mathcal{A}_{\perp \mu}^{\bar{n}}$.

The SCET matrix element of this operator can be written as

$$
\begin{aligned}
\left\langle p^{\prime}\left|\mathcal{A}_{\perp \mu}^{n} \mathcal{A}_{\perp \nu}^{\bar{n}}\right| p\right\rangle_{\mathrm{SCET}}= & g_{\alpha \beta}^{\perp} \bar{N}_{n} 1 N_{\bar{n}} \mathcal{F}^{g}(t) \\
& +\epsilon_{\alpha \beta}^{\perp} \bar{N}_{n} i \gamma_{5} N_{\bar{n}} \tilde{\mathcal{F}}^{g}(t),
\end{aligned}
$$

with

$g_{\alpha \beta}^{\perp}=g_{\alpha \beta}-\frac{1}{2}\left(n^{\alpha} \bar{n}^{\beta}+\bar{n}^{\alpha} n^{\beta}\right)$,

$\epsilon_{\alpha \beta}^{\perp}=\frac{1}{2} \epsilon_{\alpha \beta \rho \sigma} n^{\rho} \bar{n}^{\sigma}$.

In SCET-II, the contribution of each collinear sector yields a soft-collinear operator at least of order $\lambda^{7}$ :

$$
\begin{aligned}
T & \left\{\mathcal{A}_{\perp \mu}^{n}, \mathcal{L}^{(1, n)}\left[\bar{\xi} A_{\perp} q\right], \mathcal{L}^{(1, n)}[\bar{\xi}(n \cdot A) \xi], \mathcal{L}^{(2, n)}\right. \\
& {\left.\left[\bar{\xi}^{c}(\bar{n} \cdot A) A_{\perp} q\right], \mathcal{L}_{\text {int }}^{(2, n)}\left[\bar{\xi}^{c} A_{\perp} q\right]\right\} \sim O_{n}^{(6)} * J_{n} * q q q, }
\end{aligned}
$$

where $J_{n}$ is the hard-collinear kernel (jet function) and the asterisks denotes the appropriate convolutions. The SCET interactions $\mathcal{L}_{\text {int }}^{(i, n)}$ are shown schematically. ${ }^{1}$ The $T$-product in Eq. (32) can be illustrated with the help of the Feynman diagrams in Fig. 1. A similar $T$-product also describes the second collinear sector. Notice that the collinear operators in this case are the leading-order operators. Nevertheless, the helicity-flip structure of the amplitude is provided by the chiral-odd three-quark soft correlator. The total contribution associated with the operator (28) is of order $\lambda^{14}$ as required. However, the hard coefficient function of the gluon operator (28) is subleading in $\alpha_{s}$. In our further analysis, we restrict our consideration to the leading-order accuracy in the hard coupling $\alpha_{s}$. Therefore we neglect the contribution of the pure gluonic operator (28).

The other suitable operators $\mathcal{O}_{I}$ are of order $\lambda^{3}$ and can be built from the quark-gluon combinations $\bar{\chi}_{n}(0) \gamma_{\perp}^{\alpha} \mathcal{A}_{\perp \beta}^{n}$ $(\lambda \bar{n}) \chi_{\bar{n}}(0)$ and $\bar{\chi}_{n}(0) \gamma_{\perp}^{\alpha} \mathcal{A}_{\perp \beta}^{\bar{n}}(\lambda n) \chi_{\bar{n}}(0)$. We find the following two relevant scalar operators:

$$
O_{q}^{(3)}(\lambda)=\bar{\chi}_{n}^{q}\left\{\mathcal{A}_{\perp}^{n}+\mathcal{A}_{\perp}^{\bar{n}}\right\} \chi_{\bar{n}}^{q}+\chi_{\bar{n}}^{q}\left(\mathcal{A}_{\perp}^{\bar{n}}+\mathcal{A}_{\perp}^{n}\right) \chi_{n}^{q},
$$

$\tilde{O}_{q}^{(3)}(\lambda)=\bar{\chi}_{n}^{q}\left\{\tilde{\mathcal{A}}_{\perp}^{n}-\tilde{\mathcal{A}}_{\perp}^{\bar{n}}\right\} \chi_{\bar{n}}^{q}+\chi_{\bar{n}}^{q}\left(\tilde{\mathcal{A}}_{\perp}^{\bar{n}}-\tilde{\mathcal{A}}_{\perp}^{n}\right) \chi_{n}^{q}$,

\footnotetext{
${ }^{1}$ The explicit expressions can be found in Ref. [16].
} 
where the index $q$ denotes the quark flavor and

$\tilde{\mathcal{A}}_{\perp \alpha}^{n}=\epsilon_{\perp}^{\alpha \beta} \mathcal{A}_{\perp \beta}^{n}$.

The higher-order subleading operators of this type can be constructed adding the gluon fields $A_{\perp} \sim \lambda$ or $\left(A^{n} \cdot n\right) \sim \lambda^{2}$. Such operators will be suppressed as $\mathcal{O}\left(\lambda^{5}\right)$. We find that in SCET-II these operators provide the power suppressed contributions $\sim \mathcal{O}\left(\lambda^{16}\right)$ and therefore can be neglected. We shall not provide a proof of this statement in the present work and accept it as a plausible working assumption. Then at leading order in the hard coupling $\alpha_{S}$ the power suppressed helicityflip contribution is only described by the two operators $O_{q}^{(3)}$ and $\tilde{O}_{q}^{(3)}$.

In order to show the relevance of the SCET-I operators let us demonstrate the mixing of the soft-spectator contributions described by the operators (33) and (34) with the hard-spectator configuration. Such a mixing is provided by the appropriate hard-collinear $T$-products which describe the matching on the SCET-II soft-collinear operators. In order to simplify this discussion we consider the contractions of the hard-collinear fields in each hard-collinear sector separately (the collinear and soft fields are considered as external)

$T\left\{O_{q}^{(3)}\right\}=T\left\{\bar{\chi}_{n} \mathcal{A}_{\perp}^{n}\right\} T\left\{\chi_{\bar{n}}\right\}$.

The total soft-collinear operator is given by the suitable softcollinear combinations from each hard-collinear sector. The $T$-product of the hard-collinear field $\chi_{n, \bar{n}}$ can be interpreted as a transition of the hard-collinear quark and two softspectator quarks into three collinear quarks or vice versa, schematically

$T\left\{\chi_{\bar{n}}\right\} \simeq \bar{q} \bar{q} * J_{\bar{n}} * O_{\bar{n}}^{(6)} \sim \lambda^{6}$.

A combination of such $T$-products yields the soft-collinear operator describing the soft-spectator contribution for the leading amplitudes $T_{2,4,6}$; see details in Ref. [16]. The configurations with the subleading collinear operators can be generated from the hard-collinear sub-operator $\bar{\chi}_{n} \mathcal{A}_{\perp}^{n}$ in Eq. (36). For instance, matching on a twist- 4 collinear operator $O_{n}^{(8)} \sim \bar{\xi}_{n}^{c} \bar{\xi}_{n}^{c} \bar{\xi}_{n}^{c} A_{\perp c}^{n}$ can be described by the following $T$-products:

$$
\begin{aligned}
& T\left\{\bar{\chi}_{n}^{c} \mathcal{A}_{\perp}^{n}, \mathcal{L}_{\mathrm{int}}^{(1, n)}\left[\bar{\xi} A_{\perp} q\right], \mathcal{L}_{\mathrm{int}}^{(2, n)}\left[\bar{\xi}^{c} A_{\perp}^{c} A_{\perp} \xi\right], \mathcal{L}_{\mathrm{int}}^{(2, n)}\left[\bar{\xi}^{c} A_{\perp} q\right]\right\} \\
& \quad \sim O_{n}^{(8)} * J_{n} * q q \sim \lambda^{8},
\end{aligned}
$$

$$
\begin{aligned}
T & \left\{\bar{\chi}_{n} \mathcal{A}_{\perp c}^{n}, \mathcal{L}_{\text {int }}^{(1, n)}\left[\bar{\xi}^{c}(n \cdot A) \xi\right], \mathcal{L}_{\text {int }}^{(2, n)}\left[\bar{\xi}^{c}(\bar{n} \cdot A) A_{\perp} q\right],\right. \\
& \left.\mathcal{L}_{\text {int }}^{(2, n)}\left[\bar{\xi}^{c} A_{\perp} q\right]\right\} \sim O_{n}^{(8)} * J_{n} * q q \sim \lambda^{8} .
\end{aligned}
$$

The diagrams described by these $T$-products are shown in Fig. 1b, c, respectively. We also accept that the collinear fields which appear in the SCET-I operators in Eqs. (38) and (39) are generated by the substitution $\phi_{h c} \rightarrow \phi_{h c}+\phi_{c}$ performing matching onto SCET-II operators. Combining results of the two hard-collinear $T$-products one obtains a soft-collinear operator

$T\left\{O_{q}^{(3)}\right\} \simeq O_{n}^{(8)} * J_{n} * q q \bar{q} \bar{q} * J_{\bar{n}} * O_{\bar{n}}^{(6)} \sim \lambda^{14}$,

which consist of the same collinear operators as the appropriate hard-spectator contribution $O_{n}^{(8)} * \tilde{T} * O_{\bar{n}}^{(6)}$. Here we will not study the structure of all possible collinear contributions. We expect that the two presented examples clearly illustrate the presence of the soft-spectator contributions in Eq. (22). In the following discussion we assume that at the leading order in $\alpha_{s}$ the soft-spectator contribution is only described by the matrix elements of the two operators (33) and (34).

Let us consider SCET matrix elements of these operators. They can be described as

$$
\begin{aligned}
& \left\langle p^{\prime}\left|\sum_{q=u, d} e_{q}^{2} O_{q}^{(3)}(\lambda)\right| p\right\rangle_{\text {SCET }} \\
& =m \bar{N}_{n} 1 N_{\bar{n}} \int_{0}^{1} \mathrm{~d} \tau\left\{e^{i \lambda\left(p^{\prime} \cdot \bar{n}\right) \tau}+e^{-i \lambda(p \cdot n) \tau}\right\} \mathcal{G}(\tau, t), \\
& \left\langle p^{\prime}\left|\sum_{q=u, d} e_{q}^{2} \tilde{O}_{q}^{(3)}(\lambda)\right| p\right\rangle_{\text {SCET }} \\
& =m \bar{N}_{n} \gamma_{5} N_{\bar{n}} \int_{0}^{1} \mathrm{~d} \tau\left\{e^{i \lambda\left(p^{\prime} \cdot \bar{n}\right) \tau}+e^{-i \lambda(p \cdot n) \tau}\right\} \tilde{\mathcal{G}}(\tau, t),
\end{aligned}
$$

where on the $l h s$ we defined the required flavor combinations. Dimensionless amplitudes $\mathcal{G}$ and $\tilde{\mathcal{G}}$ also depend on the factorization scale $\mu_{F}$ which is not shown for simplicity. This scale separates contributions from the hard and hard-collinear regions. The SCET-I amplitudes describes the dynamics associated with hard-collinear scale $\sim \sqrt{\Lambda Q}$ and soft scale $\sim \Lambda$. Therefore these amplitudes are functions of the momentum transfer. The fraction $\tau$ can be interpreted as the fraction of the collinear momentum carried by the hardcollinear transverse gluon.

In order to obtain a formal factorization formula for the amplitudes $T_{1,3,5}$ one has to take the matrix element from Eq. (22) and use for the soft-spectator contributions on the rhs the matrix elements defined in Eqs. (25), (42), and (41). On the other hand, the nucleon spinors in the parametrization (7) appearing on the lhs must be rewritten in terms of the large components defined in (26).

For illustration let us consider the calculation of amplitudes $T_{1,2}$. These amplitudes can easily be singled out using the contraction

$$
\begin{aligned}
-\mathcal{T}_{12}^{\mu \nu} \bar{N}\left(p^{\prime}\right) \mathcal{A}^{\mu \nu} N(p)= & \bar{N}\left(p^{\prime}\right)\left(T_{1}+\not K T_{2}\right) N(p) \\
= & -\mathcal{T}_{12}^{\mu \nu}\left\langle p^{\prime}\right| \sum \tilde{C}^{\mu \nu} * \mathcal{O}_{I} \\
& +\sum O_{n}^{(i)} * \tilde{T}^{\mu \nu} * O_{\bar{n}}^{(j)}|p\rangle .
\end{aligned}
$$


The lhs can be rewritten as

$$
\begin{aligned}
\bar{N}\left(p^{\prime}\right)\left(T_{1}+\not K T_{2}\right) N(p)= & \bar{N}_{n} 1 N_{\bar{n}}(1+\kappa) \\
& \times\left\{T_{1}+\frac{m(s-u)}{4 m^{2}-t} T_{2}\right\} \\
& +\bar{N}_{n} q_{\perp} N_{\bar{n}}(1-\kappa) T_{2},
\end{aligned}
$$

where we used

$\bar{N}\left(p^{\prime}\right) \not K N(p)=\bar{N}_{n} q_{\perp} N_{\bar{n}}(1-\kappa)+\bar{N}_{n} 1 N_{\bar{n}} \frac{m}{W} K \cdot(n+\bar{n})$,

$\bar{N}\left(p^{\prime}\right) 1 N(p)=\bar{N}_{n} 1 N_{\bar{n}}(1+\kappa)$.

The rhs of (43) can be written as

$$
\begin{gathered}
-\mathcal{T}_{12}^{\mu \nu}\left\langle p^{\prime}\left|\sum \tilde{C}^{\mu \nu} * \mathcal{O}_{I}+\sum O_{n}^{(i)} * \tilde{T}^{\mu \nu} * O_{\bar{n}}^{(j)}\right| p\right\rangle \\
=\bar{N}_{n} q_{\perp} N_{\bar{n}}\left\{C_{2}(s, t) \mathcal{F}_{1}+\Psi_{\mathrm{tw} 3} * H_{2}(s, t) * \Psi_{\mathrm{tw} 3}\right\}
\end{gathered}
$$

$+\bar{N}_{n} 1 N_{\bar{n}}\left\{m C_{1}(s, t) * \mathcal{G}(t)+\Psi_{\mathrm{tw} 3} * H_{1}(s, t) * \Psi_{\mathrm{tw} 4}\right\}+\cdots$

Here $C_{1,2}$ and $H_{1,2}$ denote the momentum space hard coefficient functions in the soft- and hard-spectator contributions, respectively. The asterisks denote the convolution integrals with respect to the collinear fractions, the hard-spectator contributions are shown schematically, $\Psi_{\mathrm{tw} 3}, \Psi_{\mathrm{tw} 4}$ denote the nucleon distribution amplitudes of twist-3 and twist-4, respectively.

Comparing Eqs. (44) and (47) one obtains

$$
\begin{aligned}
T_{2} \simeq & (1-\kappa)^{-1}\left\{C_{2}(s, t) \mathcal{F}(t)+\Psi_{\mathrm{tw} 3} * H_{2}(s, t) * \Psi_{\mathrm{tw} 3}\right\}, \\
T_{1} \simeq & -\frac{m(s-u)}{4 m^{2}-t} T_{2}+(1+\kappa)^{-1}\left\{m C_{1}(s, t) * \mathcal{G}(t)\right. \\
& \left.+\Psi_{\mathrm{tw} 3} * H_{1}(s, t) * \Psi_{\mathrm{tw} 4}\right\} .
\end{aligned}
$$

Using Eq. (11) one also finds

$$
\bar{T}_{1} \simeq(1+\kappa)^{-1}\left\{m C_{1}(s, t) * \mathcal{G}(t)+\Psi_{\mathrm{tw} 3} * H_{1}(s, t) * \Psi_{\mathrm{tw} 4}\right\} .
$$

This clarify the substitution introduced in Eq. (11): such redefinition removes the kinematical part associated with $T_{2}$ from the expression for $T_{1}$ in Eq. (50). The soft-spectator contribution of the amplitude $\bar{T}_{1}$ is only defined by the subleading SCET amplitude $\mathcal{G}(\tau, t)$. We also keep the power suppressed factors $(1 \pm \kappa)$ in Eqs. (49)-(51) as the kinematical power corrections.
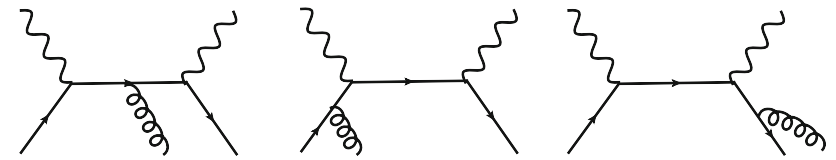

Fig. 2 The tree diagrams required for the matching onto subleading operators

Similar calculations give

$\bar{T}_{3}=(1+\kappa)^{-1}\left\{m C_{3}(s, t) * \mathcal{G}(t)+\Psi_{\mathrm{tw} 3} * H_{3}(s, t) * \Psi_{\mathrm{tw} 4}\right\}$,

$T_{4}=(1-\kappa)^{-1}\left\{C_{4}(s, t) \mathcal{F}(t)+\Psi_{\mathrm{tw} 3} * H_{4}(s, t) * \Psi_{\mathrm{tw} 3}\right\}$,

$T_{5}=(1-\kappa)^{-1}\left\{m C_{5}(s, t) * \tilde{\mathcal{G}}(t)+\Psi_{\mathrm{tw} 3} * H_{5}(s, t) * \Psi_{\mathrm{tw} 4}\right\}$,

$T_{6}=(1+\kappa)^{-1}\left\{C_{6}(s, t) \mathcal{F}(t)+\Psi_{\mathrm{tw} 3} * H_{6}(s, t) * \Psi_{\mathrm{tw} 3}\right\}$.

The hard coefficient functions $C_{2,4,6}$ can be found in Ref. [16]. The subleading coefficient functions $C_{1,3,5}$ can be computed from the diagrams in Fig. 2 and read

$C_{1}(s, t, \tau)=-\frac{1}{1-\tau} \frac{\hat{t}}{\hat{s} \hat{u}}+2\left(\frac{\hat{t}}{\hat{s} \hat{u}}+\frac{1}{\hat{t}}\right)$,

$C_{3}(s, t, \tau)=-\frac{1}{1-\tau} \frac{\hat{t}}{\hat{s} \hat{u}}-\frac{2}{\hat{t}}$,

$C_{5}(s, t, \tau)=\frac{\tau}{1-\tau} \frac{\hat{t}}{\hat{s} \hat{u}}$,

where $\tau$ is the gluon fraction, $0<\tau<1$, and the hat denotes the partonic (massless) Mandelstam variables related to the scattering angle in c.m.s. as

$\hat{t}=-\frac{\hat{s}}{2}(1-\cos \theta), \hat{u}=-\frac{\hat{s}}{2}(1+\cos \theta), \quad \hat{s}+\hat{t}+\hat{u}=0$.

To calculate the observables of Eqs. (17) and (18) the following combinations are needed:

$$
\begin{aligned}
\bar{T}_{1}-\bar{T}_{3}= & (1+\kappa)^{-1}\left\{m \Delta(s, t) \int_{0}^{1} \mathrm{~d} \tau \mathcal{G}(\tau, t)\right. \\
& \left.+\Psi_{\mathrm{tw} 3} *\left(H_{1}-H_{3}\right) * \Psi_{\mathrm{tw} 4}\right\}, \\
\bar{T}_{1}+\bar{T}_{3}= & (1+\kappa)^{-1}\left\{m \Sigma(s, t) \int_{0}^{1} \mathrm{~d} \tau \frac{\tau}{1-\tau} \mathcal{G}(\tau, t)\right. \\
& \left.+\Psi_{\mathrm{tw} 3} *\left(H_{1}+H_{3}\right) * \Psi_{\mathrm{tw} 4}\right\},
\end{aligned}
$$

where

$\Delta(s, t)=2 \frac{\hat{t}}{\hat{s} \hat{u}}\left(1+2 \frac{\hat{s} \hat{u}}{\hat{t}^{2}}\right), \quad \Sigma(s, t)=-2 \frac{\hat{t}}{\hat{s} \hat{u}}$. 
The soft- and hard-spectator contributions in the expressions for the amplitudes $T_{i}$ (49)-(55) have end-point singularities which cancel in their sum.

\section{Phenomenology}

The estimates based on the hard-spectator scattering mechanism predict an order of magnitude smaller cross section for the WACS cross section; see e.g. Refs. [6-8]. Therefore we assume that the soft-spectator contributions dominate over the hard-spectator ones in the relevant kinematical region. It is convenient to introduce the function $\mathcal{R}(s, t)$ as:

$$
\begin{aligned}
T_{2} & =C_{2}(s, t)(1-\kappa)^{-1}\left\{\mathcal{F}(t)+\Psi * \frac{H_{2}(s, t)}{C_{2}(s, t)} * \Psi\right\} \\
& \equiv C_{2}(s, t) \mathcal{R}(s, t) .
\end{aligned}
$$

In the kinematical region where the soft-spectator contribution dominates, the introduced ratio $\mathcal{R}(s, t)$ must be almost $s$-independent, i.e.

$\mathcal{R}(s, t) \simeq \mathcal{R}(t)$,

because the $s$-dependent term in Eq. (63) is only given by the hard-spectator contribution. The expressions for the other helicity-conserving amplitudes can also be defined in terms of this ratio up to small next-to-next-to-leading order corrections [16]

$$
\begin{aligned}
T_{4} & \simeq C_{4}(s, t) \mathcal{R}(t)+\mathcal{O}\left(\alpha_{s}^{2}\right), \\
T_{6} & \simeq \sqrt{\frac{-t}{4 m^{2}-t}} C_{6}(s, t) \mathcal{R}(t)+\mathcal{O}\left(\alpha_{s}^{2}\right),
\end{aligned}
$$

The similar expressions for the amplitudes $T_{2,4,6}$ have already been considered in Refs. $[15,16]$ but without the power suppressed factor $\sqrt{-t /\left(4 m^{2}-t\right)}$ in Eq. (66). This factor is part of the full kinematical power correction which was neglected in the previous work.

Deriving Eqs. (65) and (66) we use that all three amplitudes $T_{2,4,6}$ depend on the same $t$-dependent SCET amplitude $\mathcal{F}(t)$ and factorize multiplicatively. For the helicity-flip amplitudes the situation is more complicated because in this case one deals with the convolution integrals of the hard coefficient functions with two different SCET amplitudes. This leads to a more complicated structure of power suppressed contributions. In order to proceed, we introduce the three amplitudes $G_{0}(s, t), G_{1}(s, t)$, and $\tilde{G}_{1}(s, t)$ :

$$
\begin{aligned}
G_{0}(s, t)= & (1-\kappa)^{-1}\left\{\int_{0}^{1} \mathrm{~d} \tau \mathcal{G}(\tau, t)\right. \\
& \left.+\Psi_{\mathrm{tw} 3} * \frac{\left(H_{1}-H_{3}\right)(s, t)}{m \Delta(s, t)} * \Psi_{\mathrm{tw} 4}\right\},
\end{aligned}
$$

$$
\begin{aligned}
G_{1}(s, t)= & (1-\kappa)^{-1}\left\{\int_{0}^{1} \mathrm{~d} \tau \frac{\tau}{1-\tau} \mathcal{G}(\tau, t)\right. \\
& \left.+\Psi_{\mathrm{tw} 3} * \frac{\left(H_{1}+H_{3}\right)(s, t)}{m \Sigma(s, t)} * \Psi_{\mathrm{tw} 4}\right\},
\end{aligned}
$$

and

$$
\begin{aligned}
\tilde{G}_{1}(s, t)= & (1-\kappa)^{-1}\left\{\int_{0}^{1} \mathrm{~d} \tau \frac{\tau}{1-\tau} \tilde{\mathcal{G}}(\tau, t)\right. \\
& \left.+\Psi_{\mathrm{tw} 3} * \frac{H_{5}(s, t)}{C_{5}(s, t)} * \Psi_{\mathrm{tw} 4}\right\} .
\end{aligned}
$$

Analogously to $\mathcal{R}(s, t)$ these new functions are defined using the expressions for the amplitudes obtained in Eqs. (54), (60) and (61). Assuming the dominance of the soft-spectator part we again can expect that the $s$-dependence of these functions is weak

$G_{0,1}(s, t) \simeq G_{0,1}(t), \quad \tilde{G}_{1}(s, t) \simeq \tilde{G}_{1}(t)$.

Under such an assumption, we obtain

$\bar{T}_{1}-\bar{T}_{3} \simeq m \Delta(s, t) \sqrt{\frac{-t}{4 m^{2}-t}} G_{0}(t)$,

$\bar{T}_{1}+\bar{T}_{3} \simeq m \Sigma(s, t) \sqrt{\frac{-t}{4 m^{2}-t}} G_{1}(t)$,

$T_{5} \simeq m C_{5}(s, t) \tilde{G}_{1}(t)$.

Substituting the obtained expressions for the amplitudes $T_{i}$ in Eq. (13) for $W_{00}$ we obtain

$$
\begin{aligned}
W_{00} \simeq & \frac{m^{4}-s u}{4 m^{2}-t}(-t)\left\{\frac{1}{2}\left(C_{2}^{2}+C_{4}^{2}\right)+C_{6}^{2}\right\} \mathcal{R}^{2}(t) \\
& +m^{2}(-t)\left\{\Delta^{2} G_{0}^{2}(t)+\Sigma^{2} G_{1}^{2}(t)+C_{5}^{2} \tilde{G}_{1}^{2}(t)\right\} .
\end{aligned}
$$

The $r h s$ of Eq. (74) depends on the four unknown $t$-dependent functions $\mathcal{R}, G_{0,1}$, and $\tilde{G}_{1}$. Three of these functions are related to the helicity-flip amplitudes. One can expect that at large $-t$ these functions are smaller than $\mathcal{R}$. For instance, for the case of the nucleon form factors, data at large momentum transfer show that $G_{E} / G_{M} \ll 1$. Let us also assume that the helicity-flip amplitudes $G_{0,1}$ and $\tilde{G}_{1}$ in WACS are also smaller than $\mathcal{R}$. This assumption is also plausible because the amplitudes $G_{0,1}$ are defined by similar subleading operators as the form factor $G_{E}$ within the SCET formalism, see, e.g. [30]. Neglecting the helicity-flip contributions in Eq. (74) $\left(G_{0} \approx G_{1} \approx \tilde{G}_{1}=0\right.$ ) one can use the cross section data in order to extract the ratio $\mathcal{R}$ and to check the scaling behavior implied by Eq. (64). We recall that the leading-order coefficient functions $C_{2,4,6}$ read [16] 


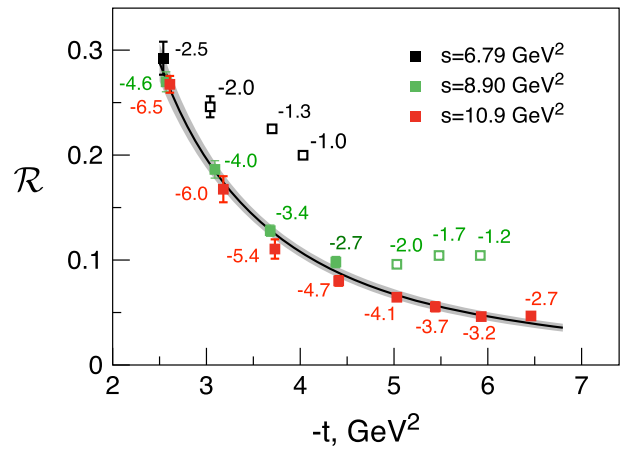

Fig. 3 Left the ratio $\mathcal{R}$ as a function of $t$. The corresponding values of $u$ are shown by the numbers next to the symbols. The open squares mark out the points with $-u<2.5 \mathrm{GeV}^{2}$. The solid line represents the empirical fit of Eq. (77), the gray bands show the $99 \%$ confidence

$C_{2}=-C_{4}=\frac{\hat{s}-\hat{u}}{\hat{s} \hat{u}}=-\frac{1}{s} \frac{3+\cos \theta}{1+\cos \theta}$,

$C_{6}=\frac{\hat{t}}{\hat{s} \hat{u}}=\frac{1}{s} \frac{1-\cos \theta}{1+\cos \theta}$.

For the scattering angle $\theta$ in Eq. (75) we use the substitution

$\cos \theta=1+\frac{2 s t}{\left(s-m^{2}\right)^{2}}$,

which also includes the power suppressed terms which are considered as a part of the kinematical corrections. The obtained results for $\mathcal{R}$ are shown in Fig. 3 .

The left plot in Fig. 3 shows the value of $\mathcal{R}$ as a function of the momentum transfer for $-t \geq 2.5 \mathrm{GeV}^{2}$. As it was assumed above, see Eq. (64), the extracted values of $\mathcal{R}$ are expected to show only a small sensitivity to $s$ when the softspectator mechanism dominates. From Fig. 3 we see that this approximate scaling behavior is observed in the region where $-u \geq 2.5 \mathrm{GeV}^{2}$. Hence we can adopt this value as a phenomenological lower limit of applicability of the described approach. For smaller values of $u$ the extracted values of $\mathcal{R}$ (shown by the open squares) demonstrate already a clear sensitivity to $s$. Thus one can observe that for $-u=1.3 \mathrm{GeV}^{2}$ $\left(-t=3.7 \mathrm{GeV}^{2}\right)$ the obtained value of $\mathcal{R}$ is about a factor 2 larger than the scaling curve. This observation clearly demonstrates that the given approach cannot describe the cross section data at small values of $u$.

The solid line in both plots in Fig. 3 corresponds to the fit of the points with $-t,-u \geq 2.5 \mathrm{GeV}^{2}$ by a simple empirical ansatz

$\mathcal{R}(t)=\left(\frac{\Lambda^{2}}{-t}\right)^{\alpha}$

where $\Lambda$ and $\alpha$ are free parameters. For their values we obtain $\Lambda=1.17 \pm 0.01 \mathrm{GeV}$ and $\alpha=2.09 \pm 0.06$. The shaded area in Fig. 3 shows the confidence interval with $\mathrm{CL}=99 \%$.

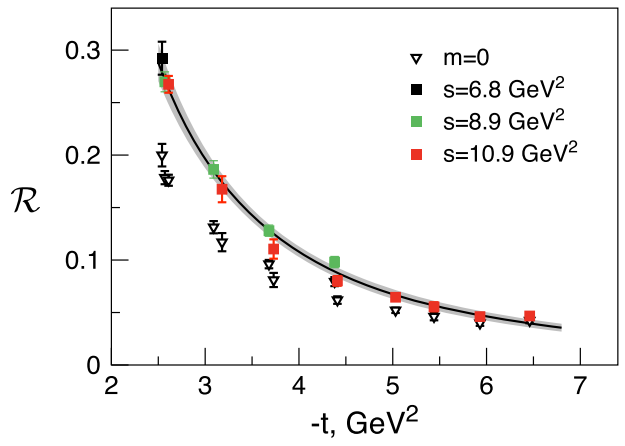

interval. Right the ratio $\mathcal{R}$ as a function of $t$, where only data with $-u>2.5 \mathrm{GeV}^{2}$ are shown. The open triangles show the values of $\mathcal{R}$ obtained without the kinematical power corrections, i.e. by setting $m=0$

On the right plot in Fig. 3 we show the effect of the kinematical power suppressed contributions. The empty triangles show the values of $\mathcal{R}$ obtained without kinematical power corrections with $m=0$. The difference between the values of $\mathcal{R}$ extracted with and without power suppressed contributions is about $30 \%$ at the lower value $-t \approx 2.5 \mathrm{GeV}^{2}$. Let us notice that the values of $\mathcal{R}$ obtained in this work are somewhat larger than ones obtained in Refs. $[15,16]$. This difference is explained by the incomplete description of the kinematical power corrections in the previous work.

The consistent results for the ratio $\mathcal{R}$, extracted in the present framework, indicate that the assumption as regards the relative smallness of helicity-flip amplitudes is probably correct. We next investigate if one can obtain an estimate of the helicity flip amplitudes from the cross section data. For this purpose, it is convenient to introduce the following ratios:

$\frac{G_{0}(t)}{\mathcal{R}(t)}=r_{0}(t), \quad \frac{G_{1}(t)}{\mathcal{R}(t)}=r_{1}(t), \quad \frac{\tilde{G}_{1}(t)}{\mathcal{R}(t)}=\tilde{r}_{1}(t)$.

In the following discussion we assume that numerically these three quantities are of the same order and small

$\left|r_{0}\right| \sim\left|r_{1}\right| \sim\left|\tilde{r}_{1}\right|<1$.

In order to see the relevance of the different subleading contributions let us consider the following ratio of the cross sections at $s=8.9 \mathrm{GeV}^{2}$ and $-t=2.5 \mathrm{GeV}^{2}$, which can be expressed as

$$
\begin{aligned}
\frac{\mathrm{d} \sigma\left(r_{0}, r_{1}, \tilde{r}_{1}\right)}{\mathrm{d} t} / \frac{\mathrm{d} \sigma(0,0,0)}{\mathrm{d} t}= & 1+2.08 r_{0}^{2}+0.02 r_{1}^{2} \\
& +0.005 \tilde{r}_{1}^{2}
\end{aligned}
$$

One can see that the largest numerical impact is provided by the contribution proportional to $r_{0}$, the other two contributions in Eq. (80) have very small coefficients and 


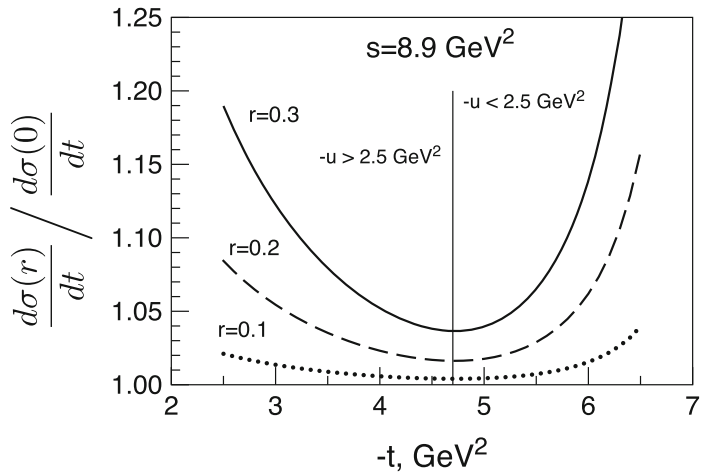

Fig. 4 The ratio of the cross sections as a function of momentum transfer at different values of $r_{0}=r_{1}=\tilde{r}_{1}=r$

therefore their numerical impact is negligible. This observation also remains valid for other values of $s$ in the region $-t,-u \geq 2.5 \mathrm{GeV}^{2}$. In Fig. 4 we show the cross section ratio of Eq. (80) at $s=8.9 \mathrm{GeV}^{2}$ as a function of momentum transfer. For simplicity we take the same values for all ratios, i.e. $r_{0}=r_{1}=\tilde{r}_{1}=r$.

We can see that correction from the helicity-flip contributions are largest at small $-t$ and smallest at the boundary where $-u \simeq 2.5 \mathrm{GeV}^{2}$. For illustration we also show the backward region where $-u \leq 2.5 \mathrm{GeV}^{2}$ and our description is not applicable. One can see that in this region the contribution of the subleading amplitudes grows and becomes more and more important. This can also be understood from Eq. (74). The kinematical coefficient in front of $\mathcal{R}^{2}$ disappears in the backward region because $\left(m^{4}-s u\right) \rightarrow 0$. Due to the relative smallness of the contribution proportional to $\mathcal{R}^{2}$ in the cross section at small $-u$, the helicity-flip terms become more important.

The relative smallness of the contributions with unknown $r_{1}$ and $\tilde{r}_{1}$ allows one to exclude them from the consideration and perform an analysis of the cross section data in order to extract the values of $\mathcal{R}(t)$ and to constrain $G_{0}(t)$. Each data point provides an inequality $\mathrm{d} \sigma_{\min } \leq \alpha \mathcal{R}^{2}+\beta G_{0}^{2} \leq \mathrm{d} \sigma_{\max }$ where $\mathrm{d} \sigma_{\max , \min }=\mathrm{d} \sigma \pm \Delta$ is the maximal and minimal experimental values of the cross section and $\alpha, \beta$ are known coefficients. In order to find the restrictions on two unknown quantities $\mathcal{R}^{2}$ and $G_{0}^{2}$ one needs at least two data points at the same $t$ and different $s$. The largest effect from $G_{0}$ is expected at small momentum transfer; see Fig. 4. Therefore we consider three data points at $-t \simeq 2.5 \mathrm{GeV}^{2}$ and $s=6.8,8.9,10.9 \mathrm{GeV}^{2}$ that provide us with three couples of inequalities. Combining the constraints from each set of inequalities we obtain the following restrictions: $\mathcal{R}=$ $0.273-0.279$ and $G_{0}=0.0-0.045$. The obtained value of $\mathcal{R}$ is within the confidence interval shown in Fig. 3. This results allows us to estimate the upper bound for the ratio $r_{0}$

$\left|r_{0}\left(-t=2.5 \mathrm{GeV}^{2}\right)\right| \leq 0.16$.

From Fig. 4 it is also seen that in this case the contribution to the cross section provided by $r_{0}$ is below $10 \%$. Such uncertainty is comparable with the theoretical uncertainties such as next-to-leading corrections or the hard-spectator corrections. Hence the result (81) must be understood as a qualitative estimate.

Let us study the effect of subleading amplitudes in the asymmetries described in Sect. 2. The asymmetries $K_{\mathrm{LL}}$ and $K_{\mathrm{LS}}$ have already been measured at JLab in two experiments: for large $-t$ but relatively small $-u=1.1 \mathrm{GeV}^{2}[3]$ and in the more appropriate kinematical region for the present work [31] (the latter analysis is not yet completed). One more experiment has recently been suggested in order to measure the initial state helicity correlation $A_{\mathrm{LL}}$ in WACS [32].

As we concluded above the presented approach is not applicable in the region of small $-u<2.5 \mathrm{GeV}^{2}$. Hence we cannot use it in order to describe asymmetries presented in Ref. [3]. Therefore despite the numerical results obtained in Ref. [16], agreement with $K_{\mathrm{LL}}$ should only be interpreted as qualitative. However, the here obtained results can be used for estimates of the asymmetries in the other experiments with more suitable kinematics; see Table 1.

Using the leading-order expressions (63)-(66) and (75) the different combinations of the amplitudes appearing in Eqs. (17), (18) can be presented as follows:

$$
\begin{aligned}
& \left(T_{2}-T_{4}\right) T_{6}^{*} \simeq 2 C_{2} C_{6} \sqrt{\frac{-t}{4 m^{2}-t}} \mathcal{R}^{2}(t), \\
& \left(T_{2}+T_{4}\right) \bar{T}_{5}^{*} \sim \mathcal{O}\left(\alpha_{s}\right) \approx 0 .
\end{aligned}
$$

Using Eqs. (71), (72), and (73) we also obtain

$$
\begin{aligned}
& \left(\bar{T}_{3}-\bar{T}_{1}\right) T_{6}^{*} \simeq m \Delta C_{6} \frac{-t}{4 m^{2}-t} G_{0}(t) \mathcal{R}(t), \\
& \left(\bar{T}_{3}+\bar{T}_{1}\right) T_{5}^{*} \simeq m^{2} \Sigma C_{5} \sqrt{\frac{-t}{4 m^{2}-t}} G_{1}(t) \tilde{G}_{1}(t) .
\end{aligned}
$$

\begin{tabular}{|c|c|c|c|c|c|c|c|}
\hline \multicolumn{4}{|c|}{$K_{\mathrm{LL}}$ at $s=9 \mathrm{GeV}^{2}$, Ref. [31] } & \multicolumn{4}{|c|}{$A_{\mathrm{LL}}$ at $s=8 \mathrm{GeV}^{2}$, Ref. [32] } \\
\hline$\theta$ & $70^{\circ}$ & $90^{\circ}$ & $110^{\circ}$ & $\theta$ & $60^{\circ}$ & $90^{\circ}$ & $136^{\circ}$ \\
\hline$-t, \mathrm{GeV}^{2}$ & 2.4 & 3.6 & 4.9 & $-t, \mathrm{GeV}^{2}$ & 1.6 & 3.1 & 5.4 \\
\hline$-u, \mathrm{GeV}^{2}$ & 4.8 & 3.5 & 2.3 & $-u, \mathrm{GeV}^{2}$ & 4.6 & 3.0 & 0.8 \\
\hline
\end{tabular}

Table 1 The kinematical regions in the two experiments of Refs. [31,32] 
From the given expressions one can easily observe that the contribution proportional to $r_{0}$ appears in the numerator of all asymmetries and therefore one can expect that these observables can be more sensitive to this subleading amplitude. By evaluating these asymmetries at $-t=2.5 \mathrm{GeV}^{2}$, we obtain

$$
\begin{aligned}
& K_{\mathrm{LL}} {\left[s=9 \mathrm{GeV}^{2}, \theta=71.5^{\circ}\right] } \\
&= \frac{0.46+0.27 r_{0}+0.01 r_{1} \tilde{r}_{1}}{1.47+3.1 r_{0}^{2}+0.03 r_{1}^{2}+0.007 \tilde{r}_{1}^{2}} \\
& \approx \frac{0.46+0.27 r_{0}}{1.47+3.1 r_{0}^{2}}, \\
& K_{\mathrm{LS}}\left[s=9 \mathrm{GeV}^{2}, \theta=71.5^{\circ}\right] \\
&= \frac{0.36-0.34 r_{0}+0.009 r_{1} \tilde{r}_{1}}{1.47+3.1 r_{0}^{2}+0.03 r_{1}^{2}+0.007 \tilde{r}_{1}^{2}} \\
& \approx \frac{0.36-0.34 r_{0}}{1.47+3.1 r_{0}^{2}}, \\
& A_{\mathrm{LL}}\left[s=8 \mathrm{GeV}^{2}, \theta=78^{\circ}\right] \\
&=\frac{0.56+0.28 r_{0}-0.02 r_{1} \tilde{r}_{1}}{1.49+2.7 r_{0}^{2}+0.06 r_{1}^{2}+0.01 \tilde{r}_{1}^{2}} \\
& \approx \frac{0.56+0.28 r_{0}}{1.49+2.7 r_{0}^{2}} .
\end{aligned}
$$

We again observe that the contributions proportional to $r_{1}$ and $\tilde{r}_{1}$ are practically negligible. In this case, all three asymmetries depend on the same unknown quantity $r_{0}$ at fixed momentum transfer. Assuming that $r_{0}$ is restricted as in (81) we find

$K_{\mathrm{LL}}\left[s=9, \theta=71.5^{\circ}\right]=0.31_{-0.04}^{+0.01}$,

where the central number is computed at $r_{0}=0$. The uncertainty in (89) is smaller than the estimated statistical accuracy \pm 0.06 in this experiment [31]. It is natural to expect that $K_{\mathrm{LS}}$ is more sensitive to the value $r_{0}$ because in this observable helicity-flip contributions are not power suppressed. Using (87) we find

$$
K_{\mathrm{LS}}\left[s=9, \theta=71.5^{\circ}\right]=0.24_{-0.04}^{+0.03}
$$

yielding an uncertainty of around $16 \%$ which is smaller than the expected statistical accuracy \pm 0.05 [31], for a preliminary result, see Ref. [33]. ${ }^{2}$

If we assume that in the leading-order approximation the combination $\left(T_{2}+T_{4}\right) \bar{T}_{5}^{*}$ is small, see Eq. (83), then the analytical expressions for the two asymmetries $K_{\mathrm{LL}}$ and $A_{\mathrm{LL}}$ only differ by the combination in Eq. $(85)\left(\bar{T}_{3}+\bar{T}_{1}\right) T_{5}^{*} \sim$ $r_{1} \tilde{r}_{1}$. But as one can observe from Eqs. (86) and (88) that the corresponding contribution is numerically small and therefore one obtains that $K_{\mathrm{LL}} \simeq A_{\mathrm{LL}}$. The uncertainty provided

2 The final results has been published in Ref. [34]

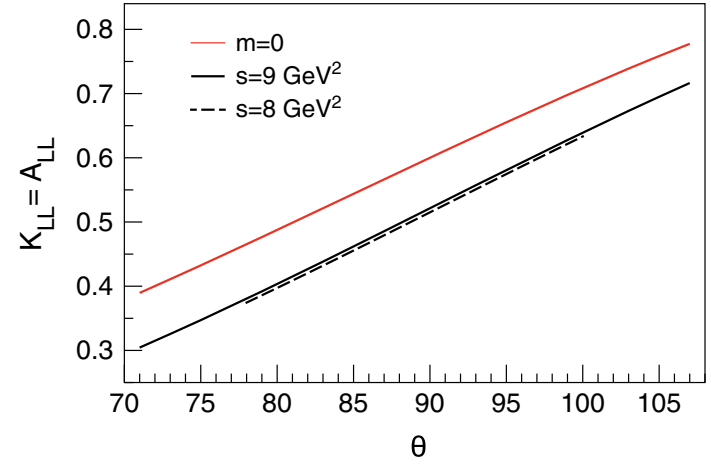

Fig. 5 The asymmetry KLL as a function of scattering angle. The red solid curve shows the result without kinematical power corrections (Klein-Nishina result). The black dashed and solid lines correspond to $s=8,9 \mathrm{GeV}^{2}$, respectively

by the ratio $r_{0}$ in $A_{\mathrm{LL}}$ in Eq. (88) yields

$A_{\mathrm{LL}}\left[s=8, \theta=78^{\circ}\right]=0.37_{-0.04}^{+0.02}$,

around $11 \%$ which is again smaller than the statistical accuracy discussed in Ref. [32].

In order to see the effect of the kinematical power corrections we plot in Fig. 5 asymmetry $K_{\mathrm{LL}}$ as a function of scattering angle for two different values of energy $s=$ $8,9 \mathrm{GeV}^{2}$ with and without power suppressed contributions. All helicity-flip contributions are taken to be zero $r_{0}=r_{1}=\tilde{r}_{1}=0$. The red line in Fig. 5 denotes the asymmetry without the power corrections which reduces to the Klein-Nishina result on the pointlike massless target $K_{\mathrm{LL}}^{K N}=\left(4-(1+\cos \theta)^{2}\right) /\left(4+(1+\cos \theta)^{2}\right)$. We only consider the angles for which $-t,-u \geq 2.5 \mathrm{GeV}^{2}$. In this region the power corrections do not change the angular dependence but reduce the value of the massless asymmetry by $25 \%$. One can also observe that the values of $K_{\mathrm{LL}}$ at both values of $s$ are almost the same. This prediction can be checked by measuring the asymmetry $A_{\mathrm{LL}}$ in the new experiment [32] at the same angles as $K_{\mathrm{LL}}$ measured in [31].

\section{Discussion}

In this work we presented a phenomenological analysis of the cross section and asymmetries of WACS in which we accounted for different power suppressed contributions. For the first time we include in the analysis the subleading helicity-flip amplitudes using the SCET framework. We assume that the dominant contribution to these amplitudes is provided by the soft-overlap configurations described by the matrix elements of SCET-I operators. We only consider the operators which appear in the leading-order approximation. The corresponding hard coefficient functions were also computed. Within this formalism we estimated the effect due to 
the power suppressed corrections in different WACS observables.

An analysis of existing cross section data allows us to conclude that the developed description can work reasonably well in the region where $-t,-u>2.5 \mathrm{GeV}^{2}$. The contribution from the helicity-flip amplitudes in the cross section is smaller than $10 \%$. We also found that the corresponding effect due to power corrections in the different asymmetries are also relatively small and to a good accuracy $A_{\mathrm{LL}}=K_{\mathrm{LL}}$ in the relevant kinematical region.

Acknowledgments This work is supported by Helmholtz Institute Mainz. N.K. is also grateful to German-US exchange program on Hadron Physics for financial support and to the staff of Old Dominion University for warm hospitality during his visit.

Open Access This article is distributed under the terms of the Creative Commons Attribution 4.0 International License (http://creativecomm ons.org/licenses/by/4.0/), which permits unrestricted use, distribution, and reproduction in any medium, provided you give appropriate credit to the original author(s) and the source, provide a link to the Creative Commons license, and indicate if changes were made. Funded by SCOAP ${ }^{3}$.

\section{References}

1. M.A. Shupe, R.H. Milburn, D.J. Quinn, J.P. Rutherfoord, A.R. Stottlemyer, S.S. Hertzbach, R.R. Kofler, F.D. Lomanno et al., Phys. Rev. D 19, 1921 (1979)

2. A. Danagoulian et al. [Hall A Collaboration], Phys. Rev. Lett. 98, 152001 (2007). arXiv:nucl-ex/0701068

3. D.J. Hamilton et al. [Jefferson Lab Hall A Collaboration], Phys. Rev. Lett. 94 (2005) 242001. arXiv:nucl-ex/0410001

4. D. Hamilton et al. [Jefferson Lab Hall C Collaboration], E1214003. Wide-angle Compton Scattering at 8 and $10 \mathrm{GeV}$ Photon Energies

5. A.S. Kronfeld, B. Nizic, Phys. Rev. D 44, 3445 (1991). [Erratumibid. D 46 (1992) 2272]

6. M. Vanderhaeghen, P.A.M. Guichon, J. Van de Wiele, Nucl. Phys. A 622, 144C (1997)

7. T.C. Brooks, L.J. Dixon, Phys. Rev. D 62, 114021 (2000). arXiv:hep-ph/0004143

8. R. Thomson, A. Pang, C.-R. Ji, Phys. Rev. D 73, 054023 (2006). arXiv:hep-ph/0602164

9. P. Kroll, M. Schurmann, W. Schweiger, Int. J. Mod. Phys. A 6, 4107 (1991)

10. A.V. Radyushkin, Phys. Rev. D 58, 114008 (1998). arXiv:hep-ph/9803316
11. M. Diehl, T. Feldmann, R. Jakob, P. Kroll, Eur. Phys. J. C 8, 409 (1999). arXiv:hep-ph/9811253

12. M. Diehl, T. Feldmann, R. Jakob, P. Kroll, Phys. Lett. B 460, 204 (1999). arXiv:hep-ph/9903268

13. M. Diehl, T. Feldmann, H.W. Huang, P. Kroll, Phys. Rev. D 67, 037502 (2003). arXiv:hep-ph/0212138

14. G.A. Miller, Phys. Rev. C 69, 052201 (2004). arXiv:nucl-th/0402092

15. N. Kivel, M. Vanderhaeghen, JHEP 1304, 029 (2013). arXiv: 1212.0683 [hep-ph]

16. N. Kivel, M. Vanderhaeghen, Nucl. Phys. B 883, 224 (2014). arXiv:1312.5456 [hep-ph]

17. D. Babusci, G. Giordano, A.I. L'vov, G. Matone, A.M. Nathan, Phys. Rev. C 58, 1013 (1998). arXiv:hep-ph/9803347

18. C.W. Bauer, S. Fleming, M.E. Luke, Phys. Rev. D 63, 014006 (2000)

19. C.W. Bauer, S. Fleming, D. Pirjol, I.W. Stewart, Phys. Rev. D 63, $114020(2001)$

20. C.W. Bauer, I.W. Stewart, Phys. Lett. B 516, 134 (2001)

21. C.W. Bauer, D. Pirjol, I.W. Stewart, Phys. Rev. D 65, 054022 (2002)

22. M. Beneke, A.P. Chapovsky, M. Diehl, T. Feldmann, Nucl. Phys. B 643, 431 (2002)

23. M. Beneke, T. Feldmann, Phys. Lett. B 553, 267 (2003)

24. A.V. Belitsky, Xd Ji, F. Yuan, Phys. Rev. Lett. 91, 092003 (2003). arXiv:hep-ph/0212351

25. B.O. Lange, M. Neubert, Nucl. Phys. B 690, 249 (2004)

26. B.O. Lange, M. Neubert, Nucl. Phys. B 723, 201 (2005). arXiv:hep-ph/0311345

27. G. Bell, T. Feldmann, Nucl. Phys. Proc. Suppl. 164, 189 (2007). arXiv:hep-ph/0509347

28. M. Beneke, L. Vernazza, Nucl. Phys. B 811, 155 (2009). arXiv:0810.3575 [hep-ph]

29. N. Kivel, Eur. Phys. J. A 48, 156 (2012). arXiv:1202.4944 [hep-ph]

30. N. Kivel, M. Vanderhaeghen, Phys. Rev. D 83, 093005 (2011). arXiv:1010.5314 [hep-ph]

31. P. Bosted et al. [Jefferson Lab Hall C Collaboration], E-07-002. Polarization transfer in Wide Angle Compton scattering

32. D. Nikolenko et al. [Jefferson Lab Hall C Collaboration], PR-1214-006. Initial State Helicity Correlation in Wide Angle Compton scattering

33. C. Fanelli, E. Cisbani, D. Hamilton, G. Salme, B. Wojtsekhowski, EPJ Web Conf. 66, 06006 (2014)

34. C. Fanelli et al, Polarization Transfer in Wide-Angle Compton Scattering and Single-Pion Photoproduction from the Proton (2015). arXiv:1506.04045 [nucl-ex] 